\title{
Brown-Peterson Metastability and the Bendersky-Davis Conjecture
}

\author{
By \\ W. Stephen WILSON*
}

Introduction

This paper represents the author's failed attempt to prove the conjectures of Bendersky and Davis, $[\mathrm{B}-\mathbb{B}]$. They apply unstable $B P$ operations to the study of nondesuspensions of truncated real projective space. As immediate corollaries they obtain nonimmersion results for real projective spaces. Unfortunately, most of their work remains conjectural. Let $\alpha(m)$ be the number of one's in the binary expansion of $m$. Strong motivation to study [B-D] is supplied by their elegant conjecture:

Conjecture $\mathbb{1}(\mathbf{s})([\mathbb{B}-\mathbb{D}])$. If $\alpha(m)=s+1$, then

$$
R P^{2(m+s)} \mp \mathbb{R}^{4 m-2 s-2}
$$

Bendersky and Davis initially proved this conjecture and all of the remaining ones for $s \leqslant 5$. Using our approach and computer tables supplied by Don Davis, we extended this to $s \leqslant 7$. However, Bendersky and Davis really did compute $s=6$, they were just overlooking a term in their preprint. Our proof of $s=7$ does not use further calculations, but is actually a theorem which depends on the $s=6$ case being true in a strong way. The cases, $s=8$ (by computation) and $s=9$ (by theory from $s=8$ ), are within reach but there is no motivation to carry out these computations. Don Davis informs us that the lowest dimensional new nonimmersion we get is for $m=493, \alpha(m)=7$, which gives $R P^{998} \nsubseteq \mathbb{R}^{1958}$, an improvement of two over $[\mathbb{A}-\mathbb{D}]$.

Let $\operatorname{gd}(E)$ be the geometric dimension of the bundle $E ; \nu_{2}(n)$, the number

Communicated by N. Shimada, October 11, 1983.

* Research Institute for Mathematical Sciences, Kyoto University, Kyoto 606, Japan. Present Address: Mathematics Department, Johns Hopkins University, Baltimore, Maryland 21218, U.S.A. 
of powers of 2 that divides $n$; $\xi_{k}$, the canonical line bundle over $R P^{k}$. Conjecture $1(s)$ is implied by Conjecture $2(s)$.

Conjecture 2(s) ([B-D]). If $d>6 s$ and $\nu_{2}\left(\begin{array}{l}a+s \\ d-s\end{array}\right)=s$, then $\operatorname{gd}\left((2 a+1) \xi_{2 d}\right)>$ $2 d-6 s-2$.

They show that this, in turn, is a direct consequence of their Conjecture 3(s). Let $R P_{k}^{n}$ be the cofiber of $R P^{k-1} \hookrightarrow R P^{n}$.

Conjecture 3(s) ([B-D]). If $d>6 s$ and $\nu_{2}\left(\begin{array}{l}a+s \\ d-s\end{array}\right)=s$, then

$$
\text { 平 } \Sigma^{2(d-a)-6 s-3} R P_{2 a+1}^{2(a+d)+1}
$$

In a difficult reduction, Bendersky and Davis deduce Conjecture 3(s) from a purely algebraic conjecture involving unstable Brown-Peterson homology information. We need some terminology. The BP cooperations are

$$
B P_{*} B P \simeq B P_{*}\left[h_{1}, h_{2}, \cdots\right], \text { where }\left|h_{n}\right|=2\left(p^{n}-1\right),
$$

and $h_{n}=c\left(t_{n}\right)$, see [A]. For $J=\left(j_{1}, j_{2}, \cdots\right)$ we write $h^{J}=h_{1}{ }^{j_{1}} h_{2}{ }^{j_{2}} \ldots$ and define the length of $J, l(J)=\sum j_{i}$. Let [2] $(x)=\sum a_{i} x^{i+1}$ be the standard two sequence. The $p=2$ reduced Brown-Peterson homology of $R P_{2 b+1}^{\infty}$ is generated as a $B P_{*}$ module by $\gamma_{k} \in B P_{2 k+1} R P_{2 b+1}^{\infty}, k \geqslant b$. The only relations are

$$
\sum_{i=0}^{k-b} a_{i} r_{k-i}=0 \quad\left(a_{0}=2, a_{1}=u_{1}\right) .
$$

Bendersky and Davis define $V_{b} \subset B P_{*} B P \otimes_{B P_{*}} B P_{*} R P_{2 b+1}^{\infty}$ as the left $B P_{*}$ module generated by all $h^{J} \otimes u \gamma_{c}$ with $2 l(J) \leqslant\left|u \gamma_{c}\right|$. Then they define

$$
W_{b} \equiv B P_{*} B P \otimes_{B P_{*}} B P_{*} R P_{2 b+1}^{\infty} / V_{b} .
$$

Note that $W_{b}$ is a left-right $B P_{*}$ module. Bendersky and Davis show that Conjecture 7(s) implies Conjecture 3(s).

Conjecture 7(s) ([B-D]). For b large $(2 b \geqslant 3 s+1)$,

$$
0 \neq 2^{s} h_{1}^{b+2 s+1} \otimes r_{b+s} \in W_{b}
$$

It is fairly easy to see that $7(s)$ implies $7(s-1)$. We show this later. The module $W_{b}$ is where Bendersky and Davis do their calculations. In fact, they set $h_{2}, h_{3}$, etc. equal to zero in $W_{b}$ and can still show $7(s)$ for $s \leqslant 6$.

Theorem 8 (Bendersky-Davis, [B-D]). Conjecture $7(s)$ is true for $s \leqslant 6$ and Conjecture $7(s) \Rightarrow$ Conjecture $3(s) \Rightarrow$ Conjecture $2(s) \Rightarrow$ Conjecture $1(s)$. 
Rather than to show directly that the elements of $W_{b}$ are nonzero, we have concentrated on describing the entire structure of $W_{b}$ through the necessary range. A few calculations make this structure clear. Its simplicity pulled the author into believing he would be able to prove the conjectures of [B-D]. Difficulties arose. The attempt to prove it almost works, but the way is blocked by one troublesome element. We describe this structure in Section 2. This leads to our version of the conjecture. We have

$$
B P_{*} \simeq Z_{(p)}\left[u_{1}, u_{2}, \cdots\right], \quad\left|u_{n}\right|=2\left(p^{n}-1\right) .
$$

As usual, [J-W], let $B P\langle 2\rangle_{*} \equiv B P_{*} /\left(u_{3}, u_{4}, \cdots\right)$. Define

$$
B^{\prime} \equiv B P\langle 2\rangle_{*} \otimes_{B P_{*}} B P_{*} B P /\left(h_{2}, h_{3}, \cdots\right) \text {. }
$$

Let $x$ have degree -2 and define, for $p=2$,

$$
B \equiv h_{1}^{-1} B^{\prime} \otimes_{B P_{*}} \widetilde{B P_{*}[[x]] /([2](x))} \text {. }
$$

Define $R \subset B$ as the left $B P\langle 2\rangle_{*}$ module generated by all negative degree elements $a \otimes c x^{i}$ with $|a| \leqslant\left|c x^{i}\right|$ except those of degree -2 with $i=1$.

Conjecture 12. $0=2(1 \otimes x) \in B / R$.

Our main result is

Theorem 13. (a) Conjecture 12 implies Conjecture 7 for all s.

(b) If $0=2(1 \otimes x) \in B / R$ modulo $\left(x^{3 k+2}\right)$, then Conjecture $7(s)$ is true for $s \leqslant$ $2 k+1$.

(c) $0=2(1 \otimes x) \in B / R$ modulo $\left(x^{13}\right)$.

Part (c) implies all of the previous conjectures for $s \leqslant 7$. It was proven by computations not represented in this paper.

In the process of our investigation, the idea of $B P$ metastability became clear and we hope it will be of independent interest. Let $\left\{B P_{n}\right\}$ be the $\Omega$ spectrum for $B P$, see $[\mathbb{R}-\mathbf{W}]$ or [W]. Using the homology suspension, we can identify $Q B P_{*} \underline{B P}_{n}$ with its image in $B P_{*} B P$. We get

$$
Q B P_{*} \underline{B P}_{2 n-1} \subset Q B P_{*} \underline{B P}_{2 n} \simeq Q B P_{*} \underline{B P}_{2 n+1} \subset Q B P_{*} \underline{B P}_{2 n+2} \subset \cdots B P_{*} B P .
$$

Theorem 15 (the metastable range). Let $M_{n} \equiv B P_{*} B P / Q B P_{*} \underline{B P}_{2 n}, n \geqslant 0$.

(a) Multiplication by $h_{1}$ defines a left-right $B P_{*}$ module map

$$
\Sigma^{2(p-1)} M_{n-1} \rightarrow M_{n},
$$


(b) which is injective for degree $s \leqslant 2\left(p^{2}-1\right) n$,

(c) and surjective for degree $s<2\left(p^{2}-1\right)(n+1)$.

$B P_{*} B P$, and thus $M_{n}$, are "sparse", i.e., they are nontrivial only in degrees which are multiples of $2(p-1)$. If we use "hyper-complex" grading (divide the real grading by $2(p-1)$ ) we can rephrase Theorem 15 as $\Sigma M_{n-1} \rightarrow M_{n}$ is an isomorphism in "hyper-complex" degrees $\leqslant(p+1) n$, which looks more like metastability. The next result is a corollary of our study of the $M_{n}$.

Corollary 16 (the metastable $\mathbb{B} \mathbb{P}_{*}$ module). Let $M \equiv \lim ^{-2(p-1) n} M_{n}$.

(a) $M$ is generated as a left $B P_{*}$ module by the $h^{J}, l(J)>0$, where $j_{1} \in Z$.

(b) The element $h^{J}$ has order $p^{l(J)}$.

(c) There is a filtration of $M$ with associated graded object $E_{0} M$, with (p) $E_{0} M=0$.

(d) $E_{0} M$ is free as a $B P_{*} /(p)$ module on the $h^{J}, l(J)>0$.

Define

$$
M_{n}(k)=M_{n} /\left(h_{1} M_{n-1}+\cdots+h_{k-1} M_{n-1}\right) .
$$

We have a generalized metastable range.

Theorem 17. Multiplication by $h_{k}$,

$$
h_{k}: \Sigma^{2\left(p^{k}-1\right)} M_{n-1}(k) \rightarrow M_{n}(k),
$$

induces a left-right $B P_{*}$ module map which is

(a) injective for degree $s<2\left(p^{k+1}-1\right) n+2\left(p^{k}-1\right)$, and

(b) surjective for degree $s<2\left(p^{k+1}-1\right)(n+1)$.

An analog of Corollary 16 is easy to state, and if true, is more difficult to prove, and, at present, of far less interest than the metastable module.

We would like to thank Martin Bendersky and Don Davis for sharing their insights into this problem. This paper owes a large debt to their work. Also, our computation of the $s=6$ and 7 cases would have been impossible without the tables supplied by Don Davis. We thank Douglas Ravenel and Hirotaka Tamanoi for many conversations about the problem. Thanks also to Professor Shimada and the RIMS, Kyoto University, for their hospitality during much of this work. We gratefully acknowledge the generous support 
of the Johns Hopkins University, the N.S.F., and Ministry of Education in Japan.

We prove our metastable results in Section 1 and we fill Section 2 with our thoughts on the Bendersky-Davis Conjectures.

\section{§1. The Metastable Range}

When we need specific generators for $B P_{*}$, we use Araki's [Ar]. Their main property is:

$$
[p](x)=\sum_{i \geqslant 0}^{F} u_{i} x^{p^{i}}=\sum_{i \geqslant 0} a_{i} x^{i+1}, \quad\left(a_{0}=p=u_{0}, a_{p-1}=u_{1}\right) .
$$

To compute the right unit we have

$$
\sum_{i, j \geqslant 0}^{R} u_{i}^{p^{j}} h_{j} x^{p^{i+j}}=\sum_{i, j \geqslant 0}^{R} h_{i}^{p^{j}} u_{j} x^{p^{i+j}}
$$

where the $R$ indicates the formal group sum with coefficients written on the right. This follows as in $[\mathbb{R}]$, see $[\mathbb{B}-\mathbb{D}]$. We use a similar notation, $L$, for the formal group law written on the left. Sometimes we use $F$ for $L$ or when there is no difference between left and right. There is a different, but equally frustrating, formula for the right unit.

Proposition 1.3. $\sum_{i \geqslant 0}^{R}\left(\sum_{j \geqslant 0}^{L} u_{j} x^{p^{j}}\right)^{p^{i}} h_{i}=\sum_{i \geqslant 0}^{R}\left(\sum_{j \geqslant 0}^{R} h_{j} x^{p^{j}}\right)^{p^{i}} u_{i}$.

Proof. We use the formula, Theorem 11.111, p. 80, of [W]. After rearranging and inserting an $x$, it reads

$$
\begin{aligned}
& \sum_{i \geqslant 0}(a(x))^{i+1} b_{i}=\sum_{i \geqslant 0}(b(x))^{i+1} a_{i} \quad \text { where } \\
& a(x)=\sum_{i \geqslant 0} a_{i} x^{i+1}=\sum_{i \geqslant 0}^{F} u_{i} x^{p^{i}}, \quad \text { and } \\
& b(x)=\sum_{i \geqslant 0} b_{i} x^{i+1}=c\left(\sum_{i \geqslant 0}^{F} t_{i} x^{p^{i}}\right)=\sum_{i \geqslant 0}^{R} h_{i} x^{p^{i}} .
\end{aligned}
$$

The result follows by substitution.

Remark. There is a misprint in 11.111. The $F$ was left out of $c\left(\sum^{F} t_{i}\right)$.

Bendersky and Davis observe that $[\mathbb{R}-\mathbb{W}]$ immediately implies:

Corollary 1.4. $Q B P_{*} \underline{B P}_{2 n} \subset B P_{*} B P$ is the left $B P_{*}$ module generated by all $h^{J} u, 2 l(J)-|u| \leqslant 2 n, u \in B P_{*}$.

We see that $Q B P_{*} \underline{B P}_{2 n} \subset B P_{*} B P$ is also a right $B P_{*}$ submodule by this. It is not really necessary to consider all of the above $h^{J} u$ because $Q B P_{*} \underline{B P}_{2 n}$ 
has been thoroughly studied. It is a free left $B P_{*}$ module and two different bases have been found. For this work we prefer Boardman's basis, ([B]),

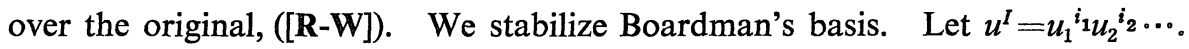

Theorem 1.5 (Boardman's basis, [B]). The left $B P_{*}$ module $Q B P_{*} \underline{B P}_{2 n} \subset$ $B P_{*} B P$ is free on generators:

$$
h^{J} u^{I}
$$

such that if

$$
I=\Delta_{k_{0}}+\Delta_{k_{1}}+\cdots+\Delta_{k_{m}}+I^{\prime}, \quad k_{0} \leqslant k_{1} \leqslant \cdots,
$$

I' a nonnegative sequence, then

$$
\begin{aligned}
& j_{m}<p^{k_{m}}, \quad m>0, \quad \text { and } \\
& 2 n-2 p^{k_{0}}<2 l(J)-\left|u^{I}\right| \leqslant 2 n .
\end{aligned}
$$

Proof. Boardman's basis is for the spaces, not after stabilization. We have elements $\bar{h}_{i} \in B P_{2 p^{i}} \underline{B P} \underline{P}_{2}, i \geqslant 0$. For $i>0, \bar{h}_{i}$ stabilizes to $h_{i}$ while $\bar{h}_{0}$ stabilizes to $1 \in B P_{*} B P$. Boardman's basis for $Q B P_{*} \underline{B P}_{2 n}$ is, where $J=\left(j_{0}, j_{1}, \cdots\right)$, $\bar{h}^{J} u^{I}$, such that if $I$ is as above, then $j_{m}<p^{k_{m}}, m \geqslant 0$, and $l(J)-\left|u^{I}\right|=2 n$. Since $\bar{h}_{0}{ }^{j_{0}}$ stabilizes to 1 , we must alter the condition on $j_{0}$ to the new condition in Theorem 1.5.

Proof of Theorem $15(a)$. Multiplication by $h_{1}$ is a left-right $B P_{*}$ module map of $B P_{*} B P$ to itself which raises degree by $2(p-1)$. Using 1.4 we see that $h_{1}$ induces a map of $Q B P_{*} \underline{B P}_{2(n-1)}$ to $Q B P_{*} \underline{B P}_{2 n}$, which are left-right $B P_{*}$ modules.

Proof of Theorem $15(b)$. Define $M_{n}^{\prime} \equiv B P_{*} B P / B P_{*}\left\{h^{J} \mid l(J) \leqslant n\right\}$. We have the commuting diagram

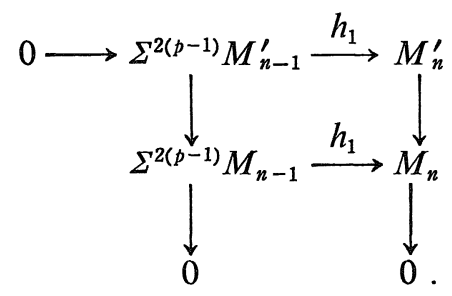

The lowest degree relation, $h^{J} u^{I}, I \neq 0$, defining $M_{n}$, which is not divisible by $h_{1}$ is, by $1.5, h_{2}^{n} u_{1}$. This is in degree $2\left(p^{2}-1\right) n+2(p-1)$. We show we have an injection for lower degree. Let $x \in M_{n-1}^{\prime}$ such that $h_{1} x$ reduces to zero in 
$M_{n}$ and is of degree such that all $h^{J} u^{I}, I \neq 0$, are divisible by $h_{1}$. Then $h_{1} x=$ $\sum a_{I J} h^{J} u^{I}$, and by injectivity $x=\sum a_{I J} h^{J-\Delta_{1}} u^{I}$ and $x$ reduces to zero in $M_{n-1}$.

Proof of Theorem 15(c). $M_{n}^{\prime}$ is generated by $h^{J}, l(J)>n$, so $M_{n}$ is also. The lowest degree $h^{J}$ with $j_{1}=0$ and $l(J)>n$ is $h_{2}^{n+1}$ in degree $2\left(p^{2}-1\right)(n+1)$. The result follows.

Proof of Theorem 17. Define $M_{n}^{\prime}(k)$ to be $M_{n}^{\prime} \mid B P_{*}\left\{h^{J} \mid j_{1}+\cdots+j_{k-1}>0\right\}$. Then the proof is the same as for 15 except that we must observe that $h_{k+1}^{n} u_{i}$ is already zero in $M_{n}^{\prime}(k)$ for $i<k$.

We can now begin a more detailed study of the metastable range.

Lemma 1.6. In degree $s \leqslant 2\left(p^{2}-1\right) n$, the left $B P_{*}$ module generators of $Q B P_{*} \underline{B P}_{2 n} \subset B P_{*} B P$ are given by all

$$
\begin{aligned}
& h^{J}, l(J) \leqslant n \quad \text { and all } \\
& h^{J} u_{i}, \quad i>0, \quad 0 \leqslant l(J)-n<p^{i} .
\end{aligned}
$$

Proof. If we take a Boardman basis element from 1.5 with two or more $u$ 's, say $h^{J} u_{k_{0}} u_{k_{1}} u^{I^{\prime}}$, then $j_{1}<p^{k_{1}}$ and our lowest degree $h^{J}$ with this a possible Boardman basis element has $h^{J}=h_{1}^{k_{1}{ }^{1}} h_{2}^{j_{2}}$, with $2 n-2 p^{k_{0}}<2 l(J)-\left|u^{I}\right| \leqslant$ $2 l(J)-\left|u_{k_{0}} u_{k_{1}}\right|=2\left(p^{k_{1}}-1\right)+2 j_{2}-2\left(p^{k_{0}}-1\right)-2\left(p^{k_{1}}-1\right)=2 j_{2}-2\left(p^{k_{0}}-1\right)$, so $2 n-2$ $<2 j_{2}$, i.e., $j_{2}=n$, but this is out of our range. So we can only have one $u$. In this case the first condition in 1.5 is empty. The second condition becomes $2 n-2 p^{i}<2 l(J)-\left|u_{i}\right| \leqslant 2 n$, which easily leads to our condition.

Definition 1.7. Order the $J$ by, $J^{\prime}<J$ if $l\left(J^{\prime}\right)<l(J)$. If $l\left(J^{\prime}\right)=l(J)$, then $J^{\prime}<J$ if $j_{k}^{\prime}>j_{k}$ when $j_{k+i}=j_{k+i}^{\prime}, i>0$. We define a filtration of $B P_{*} B P$ indexed by the $J$. Let $F_{J} B P_{*} B P$ be the left $B P_{*}$ module generated by all $h^{J^{\prime}}, J^{\prime}<J$. This induces a filtration, $F_{J} M_{n}$, of $M_{n}$. Let $E_{0} M_{n}$ denote the associated graded object. Since we will always be working in a finite range our filtration is finite.

We need a fact about the right unit.

Lemma 1.8. For $k>0, J=\left(\left(p^{k}-p\right) /(p-1), 0,0, \cdots\right), \eta\left(u_{k}\right)=(-1)^{k}\left(p h_{1}-\right.$ $\left.u_{1}\right) h_{1}^{\left(p^{k}-p\right) /(p-1)}$ modulo $\left(p^{2}, p u_{1}, F_{J} B P_{*} B P\right)$.

Proof. Let $I=\left(p, u_{1}, u_{2}, \cdots\right)$. In this degree we are working modulo $\left(I^{2}, h_{2}, h_{3}, \cdots\right)$. Modulo $I^{2}$, the formal group sums of 1.2 become ordinary sums. Modulo $\left(h_{2}, h_{3}, \cdots\right)$ the coefficient of $x^{p^{k}}$ is

$$
u_{k}=\eta\left(u_{k}\right)+h_{1}^{p^{k-1}} u_{k-1} \text { 。 }
$$


We prove the result by induction. For $k=1$ we have $\eta\left(u_{1}\right)=u_{1}-p h_{1}$. For $k>1, u_{k}$ on the left implies we are in $F_{J} B P_{*} B P$ for dimensional reasons, so our formula degenerates to $\eta\left(u_{k}\right)=-h_{1}^{p^{k-1}} u_{k-1}$. The result follows by induction.

Proposition 1.9. In degree $s \leqslant 2\left(p^{2}-1\right) n$,

(a) $p E_{0} M_{n}=0$.

(b) $E_{0} M_{n}$ is $B P_{*} /(p)$ free on the $h^{J}, l(J)>n$.

Proof. From 1.8, the defining relations for $M_{n}$, as given in 1.6, are represented in $E_{0} B P_{*} B P$ by

$$
\begin{gathered}
h^{J}, l(J) \leqslant n, \text { and }(-1)^{i} p h^{J+k_{i} \Delta_{1}} \bmod \left(p^{2}\right), \quad i>0, \\
0 \leqslant l(J)-n<p^{i},
\end{gathered}
$$

where $k_{i}=\left(p^{i}-p\right) /(p-1)+1=\left(p^{i}-1\right) /(p-1)$.

For part (a) it is enough to show that the $h^{J+k_{i} \Lambda_{1}}$ include all $h^{J^{\prime}}, l\left(J^{\prime}\right)>n$. We know that $k_{i}+p^{i}=k_{i+1}, k_{1}=1$. Find $i$ such that $k_{i} \leqslant l\left(J^{\prime}\right)-n<k_{i+1}$. Then $0 \leqslant l\left(J^{\prime}\right)-n-k_{i}<p^{i}$. We claim that $j_{1}^{\prime} \geqslant k_{i}^{\prime}$. If not, the lowest degree $h^{J^{\prime}}$ satisfying our conditions is $h_{1}^{k^{i}}{ }^{-1} h_{2}^{n+1}$ which is out of our metastable range. We can let $J=J^{\prime}-k_{i} \Delta_{1}$. This concludes part (a).

For part (b) we must show that the $J+k_{i} \Delta_{1}$ are all distinct and $l\left(J+k_{i} \Delta_{1}\right)$ $>n$. We are given that $l(J) \geqslant n$ and $k_{i}>0$, so $l\left(J+k_{i} \Delta_{1}\right)>n$. The argument in part (a) shows that the $J+k_{i} \Delta_{1}$ are all distinct.

Proposition 1.10. In degree $s \leqslant 2\left(p^{2}-1\right) n$ in $M_{n}$, the order of the generators $h^{J}, l(J)>n$, is $p^{l(J)-n}$.

Proof. We show that

$$
\begin{aligned}
& p^{l(J)-n} h^{J}=0 \text { and } \\
& p^{k} h^{J}=u_{1}^{k} h^{J-k A_{1}} \quad \text { modulo } \quad F_{J-k \Delta_{1}} M_{n}, \quad k=l(J)-n-1 .
\end{aligned}
$$

This last element is nonzero by 1.9 (b) so these prove the result. From 1.6 and 1.8 we can deduce, for arbitrary $J$, that

$$
p h^{J}=u_{1} h^{J-\Delta_{1}} \text { modulo }\left(p^{2} h^{J}, p u_{1} h^{J-\Delta_{1}}, F_{J-\Delta_{1}} M_{n}\right) .
$$

By induction on the order of $J, p^{k} F_{J-\Delta_{1}} M_{n}=0$ and $p^{k} h^{J-\Delta_{1}}=0$, so we are left with $p^{l(J)-n} h^{J}=a p^{l(J)-n+1} h^{J}$, so $p^{l(J)-n} h^{J}=0$.

By induction on $J, p^{k-1} F_{J-\Delta_{1}} M_{n} \subset F_{J-k \Delta_{1}} M_{n}, p^{k} u_{1} h^{J-\Delta_{1}}=0$, and $p^{k+1} h^{J}=0$, so 
$p^{k} h^{J}=p^{k-1}\left(p h^{J}\right)=p^{k-1}\left(u_{1} h^{J-\Delta_{1}}\right)=u_{1}\left(p^{k-1} h^{J-\Delta_{1}}\right)=u_{1}^{k} h^{J-k \Delta_{1}}$ modulo $F_{J-k \Delta_{1}} M_{n}$ by induction.

A convenient notation for $\Sigma^{-2(p-1) n} M_{n}$ in Corollary 16 is to subtract $n$ from $j_{1}$ in $M_{n}$. Corollary 16 now follows from 1.9 and 1.10 .

\section{§2. The Structure of $W_{b}$}

We want to take advantage of the Boardman basis in our analysis of $W_{b}$. In this section, $p=2$.

Lemma 2.1. $V_{b}=\sum_{c \geqslant b} Q B P_{*} \underline{B P}_{2 c+1} \otimes r_{c} \subset B P_{*} B P \bigotimes_{B P_{*}} B P_{*} R P_{2 b+1}^{\infty}$.

Proof. $Q B P_{*} \underline{B P}_{2 c+1} \simeq Q B P_{*} \underline{B P}_{2 c}$. From Corollary 1.4, this is generated as a left $B P_{*}$ module by all $h^{J} u, 2 l(J)-|u| \leqslant 2 c$, or $2 c+1$. So the above group is the left $B P_{*}$ module generated by $h^{J} u \otimes r_{c}=h^{J} \otimes u r_{c}, 2 l(J) \leqslant|u|+2 c+1=$ $\left|u r_{c}\right|$, the same as the definition of $V_{b}$ before (6) in the introduction.

Remark. Bendersky and Davis obtain their definition from this fact, their conjecture 7 takes place only in the metastable range of $Q B P_{* B P} \underline{B}_{2 c}$, so we take a limit similar to the definition of $M$ in Section 1.

The defining relations, (5), show that $\gamma_{c} \rightarrow \gamma_{c+1}$ gives an isomorphism

$$
\widetilde{B P}_{*} R P_{2(b-1)+1}^{\infty} \rightarrow \widetilde{B P}_{*} R P_{2 b+1}^{\infty} \text {. }
$$

We extend this to a left-right $B P_{*}$ map, $\Sigma^{4} W_{b-1} \rightarrow W_{b}$, given by taking $h^{J} \otimes r_{c}$ to $h^{J+\Delta_{1}} \otimes r_{c+1}$. In our range of interest we can use the results of Section 1 . This map takes generators to generators and relations among them to relations, all in a one to one fashion similar to the work in Section 1. We have an isomorphism in our range.

Define

$$
W=\lim _{b \rightarrow \infty} \Sigma^{-4 b-1} W_{b}
$$

We alter our notation so

$$
\lim _{b \rightarrow \infty} h^{J+b \Delta_{1}} \otimes r_{b+c} \equiv h^{J} \otimes r_{c}
$$

$c \geqslant 0,\left|r_{c}\right|=2 c, j_{1} \in Z$. The defining relations are now $h^{J} \otimes u r_{c}, 2 l(J) \leqslant|u|+2 c$. The lowest degree non zero element is $\lim h_{1}^{(b+1)} \otimes r_{b}=h_{1} \otimes r_{0}$, in degree 2. We can rephrase the Bendersky-Davis Conjecture 7 as.

Conjecture 2.5. $\quad 0 \neq 2^{s} h_{1}^{2 s+1} \otimes r_{s} \in W$. 
Lemma 2.6. In $W$,

$$
2^{s} h_{1}^{2 s+1} \otimes r_{s}=h_{1}^{2 s+1} \otimes u_{1}^{s} r_{0}=u_{1}^{s} h_{1}^{2 s+1} \otimes r_{0}=u_{2}^{s} h_{1} \otimes r_{0} .
$$

Remark. This shows that $7(s)$ implies $7(s-1)$.

Proof. We show that $2^{s+1} \gamma_{s}=0$, by induction on $s$, using (5).

$$
2^{s+1} \gamma_{s}=2^{s}\left(2 \gamma_{s}\right)=-2^{s}\left(\sum_{i>0} a_{i} r_{s-i}\right)=0,
$$

by induction. Now, we show $2^{s} \gamma_{s}=u_{1}^{s} \gamma_{0}$ by induction on $s$.

$$
2^{s} \gamma_{s}=2^{s-1}\left(2 \gamma_{s}\right)=-2^{s-1}\left(\sum_{i>0} a_{i} \gamma_{s-i}\right)=-2^{s-1} a_{1} \gamma_{s-1}=-a_{1} u_{1}^{s-1} \gamma_{0}
$$

Since $a_{1}=u_{1}$ and $2 \gamma_{0}=0$, we are done. This proves the first equality. Since $\eta\left(u_{1}\right)=u_{1}$ modulo (2), the second equality follows.

Modulo (2), $\eta\left(u_{2}\right)=u_{2}+u_{1} h_{1}^{2}+u_{1}^{2} h_{1}$. We show that $u_{1}^{s} h_{1}^{k} \otimes r_{0}=0, k \leqslant 2 s$. We use induction on both $s$ and $k$. If $k \leqslant 2 s-2$ we are done by induction on $s$, if $k=2 s-1$, then

$$
\left(u_{1}^{s-1} h_{1}^{2 s-3} \otimes r_{0}\right) u_{2}=u_{2}\left(u_{1}^{s-1} h_{1}^{2 s-3} \otimes r_{0}\right)+u_{1}^{s} h_{1}^{2 s-1} \otimes r_{0}+u_{1}^{s+1} h_{1}^{2 s-2} \otimes r_{0} .
$$

All terms are zero by induction except $u_{1}^{s} h_{1}^{2 s-1} \otimes \gamma_{0}$ which must therefore also be zero. The $k=2 s$ case follows similarly. We now show the last equality of 2.6 , by induction on $s$.

$$
\begin{aligned}
u_{2}^{s} h_{1} \otimes r_{0} & =u_{2}\left(u_{2}^{s-1} h_{1} \otimes r_{0}\right) \\
& =u_{2}\left(u_{1}^{s-1} h_{1}^{2 s-1} \otimes r_{0}\right) \\
& =\left(u_{1}^{s-1} h_{1}^{2 s-1} \otimes r_{0}\right) u_{2}+u_{1}^{s} h_{1}^{2 s+1} \otimes r_{0}+u_{1}^{s+1} h_{1}^{2 s} \otimes r_{0} \\
& =u_{2}^{s-1} h_{1} \otimes u_{2} r_{0}+u_{1}^{s} h_{1}^{2 s+1} \otimes r_{0}+u_{1}^{s+1} h_{1}^{2 s} \otimes r_{0} .
\end{aligned}
$$

The first term is zero because $\left|h_{1}\right| \leqslant\left|u_{2} \gamma_{0}\right|$. The last is zero by the fact that $u_{1}^{s} h_{1}^{2 s} \otimes r_{0}=0$.

Lemma 2.7. As a left $B P_{*}$ module, $W$ is generated by all $h^{J} \otimes r_{c}, l(J)>$ $c \geqslant 0, j_{1} \in Z$. The relations among these elements are given by all

$$
h^{J} \otimes u_{i} r_{c}, 0 \leqslant l(J)-c<2^{i} \text { and } h^{J} \otimes \sum_{i=0}^{c} a_{i} r_{c-i} \text {. }
$$

Proof. This follows from 1.6 and 2.1.

Definition 2.8. Let $W^{\prime}$ be the $B P_{*}$ module with generators and relations as in 2.7 except do not use the relations $h^{J} \otimes u_{1} \gamma_{c}, l(J)=c$.

We have a surjection $W^{\prime} \rightarrow W$. We believe that the elements $h^{J} \otimes u_{1} r_{c}=$ 
$u_{1}\left(h^{J} \otimes \gamma_{c}\right)+h^{J+\Delta_{1}} \otimes 2 \gamma_{c}=h^{J+\Delta_{1}} \otimes 2 \gamma_{c}, l(J)=c$, are already zero in $W^{\prime}$ and therefore that $W^{\prime} \simeq W$. If this is true, the Bendersky-Davis conjectures would follow from the next proposition because it shows that $u_{2}^{s} h_{1} \otimes r_{0}$ would be nonzero (then use 2.6).

Proposition 2.9. There is a filtration on $W^{\prime}$ with associated graded object $E_{0} W^{\prime}$ such that

(a) $\left(2, u_{1}\right) E_{0} W^{\prime}=0$, and

(b) $E_{0} W^{\prime}$ is a free $B P_{*} /\left(2, u_{1}\right)$ module on generators $h^{J} \otimes \gamma_{c}, l(J)>c \geqslant 0$.

Remark. Since $W^{\prime} \rightarrow W$ is surjective we have a similar filtration for $W$ and (a) holds.

Proof. Let $W_{1}$ be the free left $B P_{*}$ module on $h^{J} \otimes r_{c}, l(J)>c \geqslant 0$. Define a filtration by: $F_{c} W_{1}$ is the submodule generated by all $h^{J} \otimes r_{i}, i<c$. The element $h^{J} \otimes \sum a_{i} r_{c-i}$ is represented by $2\left(h^{J} \otimes r_{c}\right)$ in $E_{0} W_{1}$. Let $W_{2}$ be $W_{1}$ after setting the elements $h^{J} \otimes \sum a_{i} r_{c-i}$ equal to zero. We have a filtration $F_{c} W_{2}$. This shows that (2) $E_{0} W_{2}=0$ and $E_{0} W_{2}$ is free over $B P_{*} /(2)$ on the $h^{J} \otimes r_{c}$, $l(J)>c \geqslant 0$. We filter $E_{0} W_{2}$ by letting $F_{J} E_{0} W_{2}$ be the submodule generated by $h^{J^{\prime}} \otimes r_{c}, J^{\prime}<J$. From 2.7, the elements used to obtain $W^{\prime}$ from $W_{2}$ are the $h^{J} \otimes u_{i} r_{c}, 0 \leqslant l(J)-c<2^{i}, i>1, l(J)=c+1, i=1$. They live in $W_{2}$. Their representatives in $E_{0} E_{0} W_{2}$ are given $u_{1} h^{J+\left(2^{i}-2\right) \Delta_{1}} \otimes \gamma_{c}, 0 \leqslant l(J)-c<2^{i}, i>1$, $u_{1} h^{J} \otimes r_{c}, l(J)=c+1$. This follows from 1.8 and $2 E_{0} W_{2}=0$. Reindexing, these elements are just $u_{1} h^{J} \otimes r_{c}, l(J)>c$. Using these relations to define $W^{\prime}$ from $W_{2}$ the results, both (a) and (b), follow. (We would have some redundancy if we tried to use $h^{J} \otimes u_{1} \gamma_{c}, l(J)=c$ as well, and (b) would not follow.)

Our attention is now fixed on the elements $2 h^{J} \otimes r_{c}, l(J)=c+1$, because from the discussion before 2.9 we have:

Corollary 2.10. If, for all $l(J)=c+1,0=2\left(h^{J} \otimes r_{c}\right) \in W^{\prime}$, then $W^{\prime} \simeq W$ and conjecture 7 is true.

We now throw away all of the elements which computations have taught us are irrelevant, just as Bendersky and Davis did. Let

$$
\begin{aligned}
& \tilde{W}^{\prime} \equiv B P\langle 2\rangle_{*} \otimes_{B P_{*}} W^{\prime} /\left(h_{2}, h_{3}, \cdots\right) \\
& \tilde{W} \equiv B P\langle 2\rangle_{*} \otimes_{B P_{*}} W /\left(h_{2}, h_{3}, \cdots\right) .
\end{aligned}
$$

The following is an automatic Corollary of 2.7, 2.9 and 2.10. 
Corollary 2.12. (a) As a $B P\langle 2\rangle_{*}$ module, $\tilde{W}$ is generated by $h_{1}^{j} \otimes r_{c}$, $j>c \geqslant 0$ with relations $h_{1}^{j} \otimes u_{i} r_{c}, 0 \leqslant j-c<2^{i}$ and $h^{j} \otimes \sum_{i} a_{i} r_{c-i}$.

(b) $\tilde{W}^{\prime}$ is as in (a) but without the relations $h_{1}^{c} \otimes u_{1} \gamma_{c}=2 h_{1}^{c+1} \otimes r_{c}$.

(c) There is a filtration on $\tilde{W}^{\prime}$ such that $\left(2, u_{1}\right) E_{0} \tilde{W}^{\prime}=0$, and

(d) $E_{0} \tilde{W}^{\prime}$ is a free $Z / 2\left[u_{2}\right]$ module on $h_{1}^{j} \otimes r_{c}, j>c \geqslant 0$.

(e) If $2 h_{1}^{c+1} \otimes r_{c}=0, c \geqslant 0$, in $\tilde{W}^{\prime}$, then $\tilde{W}^{\prime} \simeq \tilde{W}$ and Conjecture 7 is true.

More is actually true, because if $2 h_{1}^{c+1} \otimes r_{c}=0$, then $2 h_{1}^{c} \otimes r_{c-1}=0$ in $\tilde{W}^{\prime}$. We prove a stronger result.

Define $\tilde{W}(k)=\tilde{W}^{\prime} /\left(2 h_{1}^{c+1} \otimes r_{c}, c<k\right) . \quad$ Then $\tilde{W}(k) \simeq \tilde{W}$ for degrees less than $\left|2 h_{1}^{k+1} \otimes r_{k}\right|$.

Proposition 2.13. If $2 h_{1}^{3 k+1} \otimes r_{3 k}=0$ in $\tilde{W}(3 k)$, then

(a) $2 h_{1}^{c+1} \otimes r_{c}=0$ in $\tilde{W}^{\prime}, c \leqslant 3 k+2$,

(b) $\quad \tilde{W}^{\prime} \simeq \tilde{W}$ in degrees less than $12(k+1)+2$, and

(c) Conjecture 7(s) is true for $s \leqslant 2 k+1$.

Remark 2.14. Hidden in this proposition is the fact that if we prove $7(s)$ for $2 k$ using our approach then it is true for $2 k+1$ as well, thus our $s=6$ implies $s=7$.

Proof. We define

$$
\beta: \Sigma^{-4} W(k) \rightarrow W(k-1), \quad \beta\left(h^{J} \otimes r_{c}\right)=h^{J-\Delta_{1}} \otimes r_{c-1}, \quad r_{-1}=0 .
$$

It is easy to verify that this is well defined. The map $\beta$ can also be defined on $\tilde{W}^{\prime}, \tilde{W}, W^{\prime}$ and $W$.

Claim. $2 h_{1}^{k+1} \otimes r_{k}=0$ in $\tilde{W}(k)$ implies $2 h_{1}^{c+1} \otimes r_{c}=0$ in $\tilde{W}^{\prime}, c \leqslant k$.

Proof of claim. We use induction on $k$. If $2 h_{1}^{k+1} \otimes r_{k}=0$ in $\tilde{W}(k)$, apply $\beta$ to deduce that $2 h_{1}^{k} \otimes r_{k-1}=0$ in $\tilde{W}(k-1)$. By induction we have $2 h_{1}^{i+1} \otimes r_{i}$ $=0$ in $\tilde{W}^{\prime}, i<k$. But now $\tilde{W}(k) \simeq \tilde{W}^{\prime}$, so $2 h_{1}^{k+1} \otimes r_{k}=0$ in $\tilde{W}^{\prime}$ as well.

Change $k$ to $3 k$ and we have proven (a) for $c \leqslant 3 k$. We define another map

$$
\alpha: \Sigma^{-2} \tilde{W}^{\prime} \rightarrow \tilde{W}^{\prime}, \quad \alpha\left(h^{J} \otimes r_{c}\right)=h^{J-\Delta_{0}} \otimes r_{c} .
$$

It is easy to show that this is well defined and exists on $\tilde{W}, W^{\prime}$, and $W$ also.

We consider $2 h_{1}^{3 k+2} \otimes r_{3 k+1}$. By $2.12(\mathrm{~d})$, 


$$
2 h_{1}^{3 k+2} \otimes r_{3 k+1}=\sum_{i, j} a_{i j} u_{2}^{k_{i j}} h_{1}^{i+j} \otimes r_{i}
$$

uniquely with $a_{i j}=0$ or 1 .

Apply $\alpha$ to both sides. The left side is zero, so the only possible nonzero coefficients on the right are $a_{i 1}$, so

$$
2 h_{1}^{3 k+2} \otimes r_{3 k+1}=\sum_{i \geqslant 0} a_{i} u_{2}^{k_{i}} h_{1}^{i+1} \otimes r_{i}
$$

Now apply $\beta$. We already have that $2 h_{1}^{3 k+1} \otimes \gamma_{3 k}=0$, so the only $a_{i}$ that can be nonzero is $a_{0}$, so

$$
2 h_{1}^{3 k+2} \otimes r_{3 k+1}=a_{0} u_{2}^{k} h_{1} \otimes r_{0}
$$

Comparing the degree of the left hand side with the right we have $12 k+6 \neq$ $6 k_{0}+2$, so $a_{0}=0$. The proof for $2 h_{1}^{3 k+3} \otimes r_{3 k+2}=0$ is the same.

Part (b) follows. Part (c) follows from (b).

Proof of Theorem 13. Define $B(k) / R \equiv B /\left(R+\left(x^{3 k+2}\right)\right)$, map

$$
B(k) / R \rightarrow \Sigma^{-(12 k+4)} \tilde{W}(3 k)
$$

by $h_{1}^{j} \otimes x^{i} \rightarrow h_{1}^{3 k+1+j} \otimes r_{3 k+1-i}$.

It is easy to check that the map is well defined. Furthermore, the map is an isomorphism in negative degrees. This is true simply because the generators and relations go to the generators and relations. It may be helpful, however, to observe first that

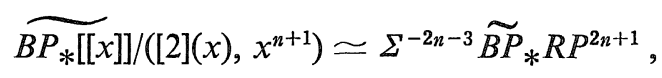

just from generators and relations.

The map takes $1 \otimes x$ to $h_{1}^{3 k+1} \otimes r_{3 k}$, so if $2(1 \otimes x)=0$, we can apply Proposition 2.13 (c) to prove part (b). Part (a) follows from (b). Part (c) is gruesome computation actually carried out modulo $x^{11}$, but the proofs of 2.13 and Theorem 13 imply that it is true modulo $x^{13}$ also.

The main differences in our approach to proving Conjecture 7 and that in $[\mathbb{B}-\mathbb{D}]$ are threefold. We like to eliminate powers of 2 . They like to eliminate powers of $u_{1}$. We like the left $B P_{*}$ module structure, they like to move things to the right. We use Boardman's basis. Bendersky and Davis told us before we began this project that the [2]-sequence played a nasty roll. The only way to approach Conjecture 12 is to write $2(1 \otimes x)=-1 \otimes \sum_{i>0} a_{i} x^{i+1}$, and it seems that we have reduced the entire problem to the nasty [2]-sequence. 
The only positive, non computational step we made towards a proof of Conjecture 12 is the following.

Proposition 2.17. In $B / R$,

$$
2(1 \otimes x)=u_{1}^{2} h_{1}^{-1} \otimes x^{2}+{ }_{R} u_{2}^{2} h_{1}^{-3} \otimes x^{4} .
$$

Remarks 2.18. The term $u_{1}^{2} h_{1}^{-1} \otimes x^{2}$ can easily be replaced with $h_{1} \otimes(2 x)^{2}$, and the [2]-sequence rears its ugly head again. If we do not tensor with $B P\langle 2\rangle_{*}$, this is

$$
2(1 \otimes x)=\sum_{i>0}^{R} u_{i}^{2} h_{1}^{1-2^{i}} \otimes x^{2^{i}}
$$

Proof. Set $h_{i}=0, i>1$ and subtract the equal terms $u_{0} y$ and $\eta\left(u_{0}\right) y ; 1.2$ becomes

$$
\sum_{i>0}^{R} u_{i} y^{2^{i}}+_{R} \sum_{i \geqslant 0}^{R} u_{i}^{2} h_{1} y^{2^{i+1}}=\sum_{i>0}^{R} \eta\left(u_{i}\right) y^{2^{i}}+_{R} \sum_{i \geqslant 0}^{R} h_{1}^{2^{i}} u_{i} y^{2^{i+1}} .
$$

The dummy variable has degree -2 . Substitute $y=\left(h_{1}^{-1} \otimes x\right)^{1 / 2}$ and we have a formula in $B / R$;

$$
\sum_{i>0}^{R} u_{i} h_{1}^{-2^{i-1}} \otimes x^{2^{i-1}}+{ }_{R} \sum_{i \geqslant 0}^{R} u_{i}^{2} h_{1}^{1-2^{i}} \otimes x^{2^{i}}=\sum_{i>0}^{R} h_{1}^{-2^{i-1}} \otimes u_{i} x^{2^{i-1}}+{ }_{R} \sum_{i \geqslant 0}^{R} 1 \otimes u_{i} x^{2^{i}} .
$$

The far right sum is $1 \otimes \sum_{i \geqslant 0}^{F} u_{i} x^{i^{i}}=1 \otimes[2](x)=0$. The terms of the other sum on the right have $\left|h_{1}^{-2^{i-1}}\right| \leqslant\left|u_{i} x^{i^{i-1}}\right|$ so they are all zero except $h_{1}^{-1} \otimes u_{1} x$, which only has one power of $x$ so it is not a given relation. The same thing occurs in the very first sum. The first term of the second sum is $4(1 \otimes x)$. Using [B-D] it is easy to see that this is zero. We have

$$
4(1 \otimes x)=2(1 \otimes 2 x)=-2\left(1 \otimes \sum_{i>0} a_{i} x^{i+1}\right) .
$$

Now, $2=-u_{1} h_{1}^{-1}+h_{1}^{-1} u_{1}$, so this is

$$
+u_{1} h_{1}^{-1} \otimes \sum_{i>0} a_{i} x^{i+1}-h_{1}^{-1} \otimes u_{1} \sum_{i>0} a_{i} x^{i+1}=0,
$$

because we never use only one power of $x$. There is some checking to do to see that the formal group sums do not alter these zeros. We have

$$
\begin{aligned}
\sum_{i>0}^{R} u_{i}^{2} h_{1}^{1-2^{i}} \otimes x^{2^{i}} & =h_{1}^{-1} \otimes u_{1} x-{ }_{R} u_{1} h_{1}^{-1} \otimes x . \\
& =\sum_{i, j} u_{1}^{j} h_{1}^{-i-j} \otimes u_{1}^{i} x^{i+j} a_{i j}^{1}, \quad \text { some } a_{i j}^{1} \in B P_{2(i+j-1)} .
\end{aligned}
$$

Again, all terms are zero except $h_{1}^{-1} \otimes u_{1} x-u_{1} h_{1}^{-1} \otimes x$ which is $u_{1} h_{1}^{-1} \otimes x+$ $2(1 \otimes x)-u_{1} h_{1}^{-1} \otimes x=2(1 \otimes x)$. 
A mindless algorithm for computing with Conjecture 12 is as follows. (1) Take the lowest power of $x$ with a non-zero coefficient. (2) Use the right unit to move $u$ 's to the left. (3) Eliminate 2 from the coefficient by use of the [2]-sequence. (4) For a term with $u_{1}$, say $a u_{1} h_{1}^{i} \otimes x^{j}, a \in B P\langle 2\rangle_{*}$, we must have $i>j$ or it is zero. Find the smallest $k$ such that $\left|h_{1}^{i-\left(2^{k}-2\right)}\right| \leqslant u_{k} x^{j}$. Then add $a h_{1}^{i-\left(2^{k}-2\right)} \otimes u_{k} x^{j}=0$ by using the right unit. From 1.8 we see that this will get rid of the term with the $u_{1}$. This is all of the algorithm, just repeat it. The reason this is all, is because, so far, we are never left with a term $u_{2}^{k} h_{1}^{i} \otimes r_{j}$. If this ever happens then our concept of $W$ is all wrong. Moreover, we have shown that if it ever happens, the first case must be $u_{2}^{2 k} h_{1}^{-3 k} \otimes x^{3 k+1}$, for some $k$. Computations can be shortened significantly by using the "excess" in [BB-D]. For example, to start, we take $2(1 \otimes x)=$ $-\sum_{i>0} 1 \otimes a_{i} x^{i+1}$. However, $2 \mid a_{2 k}$, so all terms $1 \otimes a_{2 k} x^{2 k+1}=0$ automatically.

\section{References}

[A] J.F. Adams, Quillen's work on formal groups and complex cobordism, Stable Homotopy and Generalized Homology, University of Chicago Press, Chicago, (1974), 29-120.

[Ar] S. Araki, Typical formal groups in complex cobordism and K-theory, Lectures in Math., Dept. of Math., Kyoto Univ., Tokyo, Kinokuniya Book Store, 1973.

[A-D] L. Astey and D.M. Davis, Nonimmersions of real projective spaces implied by BP, Bol. Soc. Mat. Mex., 24 (1979), 49-55.

[B-D] M. Bendersky and D.M. Davis, Unstable BP-homology and desuspensions, Amer. J. Math., to appear.

[B] J.M. Boardman, Another basis of $Q B P_{*} B P_{*}$, to appear.

[J-W] D.C. Johnson and W.S. Wilson, Projective dimension and Brown-Peterson homology, Topology, 12 (1973), 327-353.

[R] D.C. Ravenel, The structure of $B P * B P$ modulo an invariant prime ideal, Topology, 15 (1976), 149-153.

[R-W] D.C. Ravenel and W.S. Wilson, The Hopf ring for complex cobordism, J. Pure Appl. Algebra, 9 (1977), 241-280.

[W] W.S. Wilson, Brown-Peterson homology: an introduction and sampler. CBMS Regional Conference Series in Mathematics, 48, Amer. Math. Soc., Providence, 1982.

Note added in proofs. Conjecture 1 has recently been proved by Davis using Astey's direct approach. 
